\title{
IV
}

\section{DENTAL DISEASE AND ITS RELATION TO ANTISYPHILITIC THERAPY}

\author{
By LEWIS G. CRUICKSHANK, L.R.C.P., L.D.S.
}

THE following observations result from the dental examination of 400 unselected, consecutive patients (men, women and children) in all stages of syphilis under treatment, along with a like number of "controls" (patients under treatment for conditions other than syphilis). The object of the investigation was to determine the incidence of dental disease in syphilitic patients and the amount of interference with treatment resulting from oral complications.

The classification of oral sepsis was :-

(I) Dental caries.

(2) Marginal gingivitis.

(3) Pyorrhœa alveolaris. (a) Early. (b) Late.

(4) Bismuth line.

(5) Inquiry was made as to whether oral hygiene was practised or not.

A typical case of marginal gingivitis shows red, tender, congested gum margins, which may bleed easily. Such a condition, if allowed to progress, eventually leads to periodontal disease (pyorrhœea alveolaris), the gingival infection gradually spreading to involve the whole of the tooth attachments.

A case of " early" pyorrhœea shows disappearance of the interdental papilla of gum, with, perhaps, formation of calculus or " tartar" round the necks of the teeth, and early gum recession.

The typical clinical picture of advanced pyorrhœa is unmistakable : the breath is foul, the necks of the teeth exposed by destruction of the alveolar bone, and frequently covered with greenish-black calculus. The teeth are loose, and pus may be made to well up from below the gum margins on pressure.

Dental caries is of importance, as in the majority of cases it is evidence that adequate oral hygiene is not practised and may be frequently associated with abscess 


\section{DENTAL DISEASE}

formation around the apex of the tooth, giving rise to a hidden septic focus.

The classification of cases into marginal gingivitis, " early " and " late " pyorrhœa based entirely on clinical findings may be open to criticism, but it is adequate for the purpose of the present investigation. Patients whose dental condition did not fall exactly into one of these

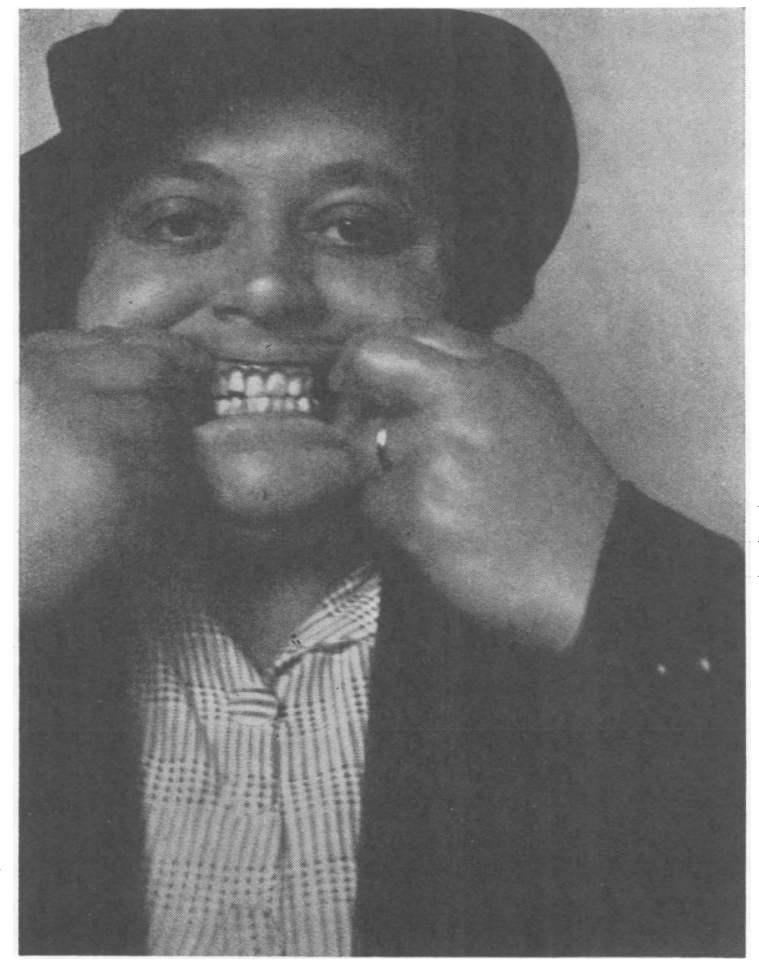

FIG. I. Early acute bismuth line, supervening on pre-existing marginal gingivitis. Condition improved rapidly with simple local measures. Bismuth recommenced in five weeks.

categories were placed in the class to which they most nearly approximated.

The results of the investigation are given below :-

Total number examined:

Syphilitic . . 400

Non-syphilitic . $\quad$. 400

Edentulous :

Syphilitic . . . $\quad$ I68 $=42 \%$

Non-syphilitic . . $\quad$ I $45=36 \%$

$28 \mathrm{I} \quad \times 2$ 


\section{BRITISH JOURNAL OF VENEREAL DISEASES}

Patients with natural teeth still present (on which the following percentages are based) :

$$
\begin{array}{ll}
\text { Syphilitic } & \cdot \quad \cdot \quad 232=58 \% \\
\text { Non-syphilitic } \quad \cdot \quad & 255=64 \%
\end{array}
$$

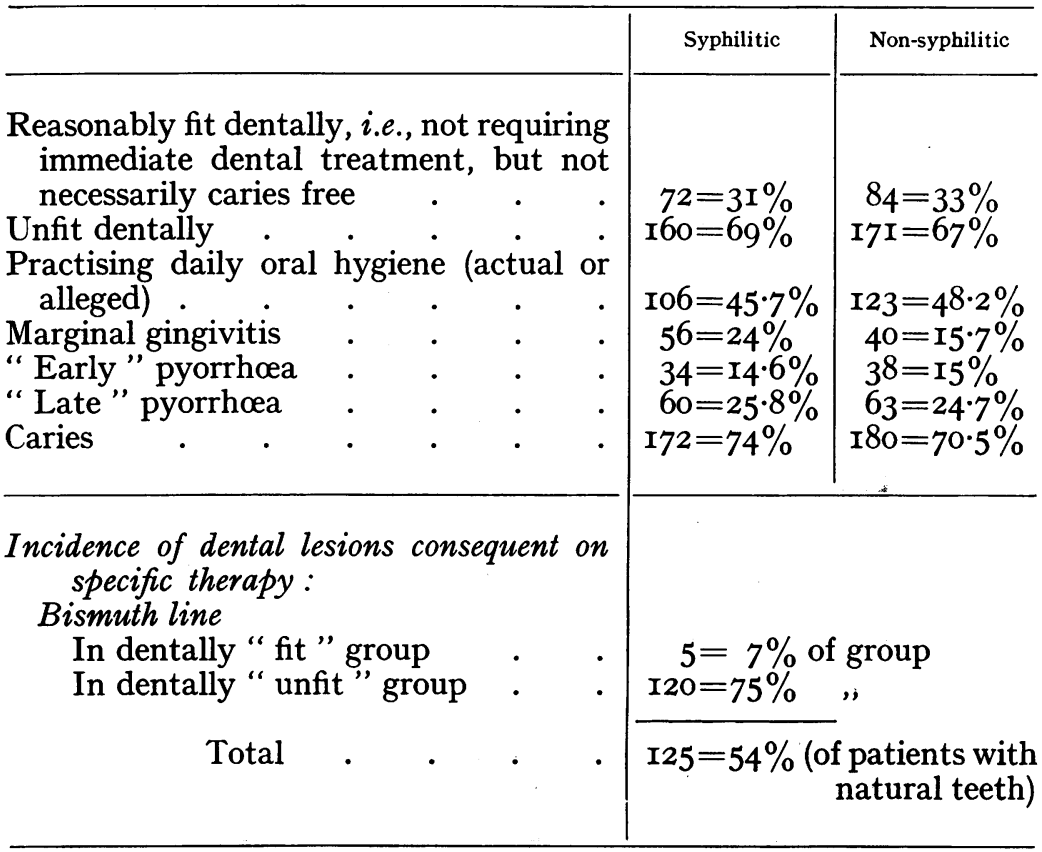

From the above figures it will be seen that the incidence of the various conditions under review is much the same for syphilitic and non-syphilitic patients. The slight balance in favour of the non-syphilitic group is probably accounted for by the fact that the average age of the group was considerably lower than that of the syphilitic group.

Only 3I per cent. of the syphilitic group could be considered reasonably healthy from the dental aspect.

The remaining 69 per cent. must be considered possible candidates for one or other of the complications of antispecific therapy attributable to the presence of dental or gingival disease.

\section{BISMUTH}

The action of bismuth on the mouth varies greatly with the individual. A " bismuth line" occurs at one time or another in practically every patient, and a mild, 


\section{DENTAL DISEASE}

symptomless, discoloration of the gum margin is not in itself a contraindication to the further employment of the drug. A bismuth line is often limited to, or more marked in, the vicinity of diseased teeth.

The generally accepted view is that the line is caused by deposition of bismuth in the form of bismuth sulphide. Histological examination of a pigmented patch shows no epidermal deposit, but that the deposit of bismuth sulphide occurs in the connective tissue cells of the dermis proper (Milian and Perin (I922), Azoulay (I922)).

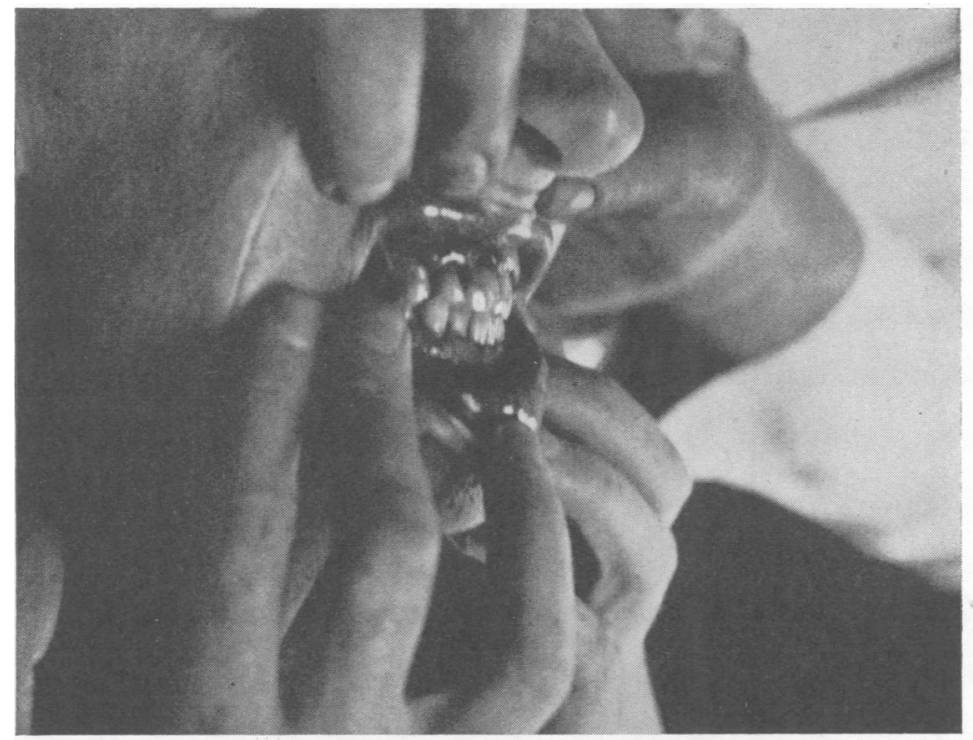

FIG. 2. Moderately severe bismuth stomatitis, with commencing gingival ulceration. Bismuth recommenced after ten weeks' local treatment.

In the presence of gingival periodontal disease, however, the bismuth line may assume a graver aspect, as in such cases the continuance of bismuth therapy may lead to ulceration and stomatitis. In most cases this ulceration involves only the gums, but more severe cases in which ulceration of the cheeks, tongue, lips and tonsils have been described (Milian and Perin (r922)).

Cases of bismuth stomatitis have been reported in which the preliminary pigmentation was absent (Buschke (I924)).

When actual ulceration does occur, the ulcers are generally of a superficial circumscribed nature, with 283 


\section{BRITISH JOURNAL OF VENEREAL DISEASES}

often a greyish-black membranous covering. There may be a pigmented areola of varying extent.

In a case recently observed following administration of a total of $\mathrm{I} \cdot 4 \mathrm{gm}$. of bismuth, the patient developed an acute ulcerative condition of the gums, involving chiefly the region of the lower anterior teeth, and the upper molars (the " tartar" areas). In the latter region there was also ulceration of the cheeks, the ulcers throughout being of the greyish, superficial type already described. There was no definite bismuth line present. Marked general malaise, fœtid breath, and extreme tenderness on mastication were present, and the patient looked " toxic." The possibility of a Vincent's infection was eliminated. Intravenous administration of calcium thiosulphate gave symptomatic relief after three to four injections of 0.6 to $0.9 \mathrm{gm}$. at daily intervals, and the local condition responded well to astringent applications, the gums having returned to normal in three to four weeks. In view of the possible ætiological relationship to bismuth, subsequent treatment was with mercury, and to date there has been no recurrence of the condition. The patient's original dental condition was one of very mild marginal gingivitis.

The bismuth line may appear as early as two weeks after the commencement of treatment, or not even after twelve to fourteen weeks of continuous bismuth therapy.

There can be little doubt that gingival and periodontal disease predispose to the early appearance of a bismuth line, and render the patient more liable to an acute bismuth stomatitis.

The duration of interruption of treatment following on oral complications of bismuth therapy varies widely for a variety of reasons, of which probably the most variable is the decision as to when a patient is fit to recommence bismuth therapy. This decision must rest with the individual clinician : no rules can be laid down.

In a series of 120 cases personally observed the average duration of interruption was found to be ten to fourteen weeks.

\section{Mercury}

Stomatitis following the use of mercurial preparations may, as in the case of bismuth, appear at almost any 


\section{DENTAL DISEASE}

time during the administration of the drug. In susceptible patients it may follow even small doses.

Excessive salivation, metallic taste in the mouth and tenderness on biting are generally the earliest symptoms. The gums become swollen and spongy, the breath fœetid, the teeth may become loose, the salivary glands enlarged. The marked salivation of mercurial stomatitis is in strong contrast to bismuth stomatitis, in which salivation is seldom in excess of normal.

The almost universal displacement of mercurial treatment by bismuth in recent years has almost completely eliminated this drug as a cause of stomatitis. A survey of case sheets of the pre-bismuth period shows, however, a comparatively small incidence of recorded oral lesions attributed to mercury.

\section{ARSENIC}

Dermatitis is one of the more serious complications of arsenical therapy in syphilis, varying in severity from a mild transient erythema to an extensive exfoliation of the skin over the whole of the body area with a danger of death from broncho-pneumonia.

Stokes and Cathcart (I923) have studied the question of focal infection in relation to arsenical dermatitis. They report one case in which the complete removal of a septic focus resulted in an almost miraculous cure of a severe dermatitis. Subsequently, however, they found that even complete extirpation of a septic focus produced no amelioration, but rather the reverse, even leading to a fatal issue. They counsel against the interference with any focus of infection during the course of an arsenical dermatitis unless it be easily accessible and completely removable at a single operation.

My personal observation of a case of severe arsenical dermatitis which cleared up with astonishing rapidity following the removal of a premolar root and drainage of the associated alveolar abscess, supports the views of Stokes and Cathcart as to the possible relationship of dental sepsis to arsenical dermatitis.

McLachlan (I937), in a series of I2 cases of arsenical dermatitis, gives an average duration of fourteen days in milder cases and twenty-two days in the more severe types. Cases in which skin residuæ have persisted for eight to ten weeks or longer are, however, not unknown. 


\section{BRITISH JOURNAL OF VENEREAL DISEASES}

The possibility of cryptic dental foci of infection adversely influencing the condition has to be borne in mind.

\section{Prophylaxis and Treatment}

A careful scrutiny of the patient's teeth and gums should be made in every case at the patient's first visit, and the necessity for strict attention to oral hygiene during treatment made clear; in the majority of cases the intelligent co-operation of the patient may be expected if adequate explanation is given. The patient's co-operation is of prime importance, and no effort should be spared to secure it.

Where any dental lesion exists arrangements should be made for its prompt treatment. There exists a common belief amongst dentists that all stages of syphilis are highly infective, and that a patient who exhibits a positive Wassermann reaction must necessarily be contagious. Such a fallacy cannot be too strongly condemned, as it may frequently constitute a great barrier to intelligent co-operation between the syphilologist and the dental surgeon. The high degree of infectivity of primary and secondary syphilis is well recognised : such cases are, however, rendered non-infectious by a comparatively small dosage of neoarsphenamine and bismuth (or mercury). Where possible such treatment should be given before the patient is referred to the dental surgeon. Tertiary syphilis may be regarded as practically noninfectious. Whilst inoculations from gummata have in some cases produced syphilis, in the vast majority of cases no infection has followed. When, however, there is doubt about the possible infectiousness of a patient requiring urgent dental treatment, the dentist should be informed of the fact and advised to wear rubber gloves.

All patients receiving bismuth (or mercury) should, apart from regular brushing of the teeth and gums with a suitable dentifrice, use a mouthwash regularly. Alum (gr. $x$. to the ounce) or common salt (teaspooinful in a tumbler of cold water) have proved very effective in practice. The latter has numerous advantages, being simple, refreshing in use, available to all, and very effective prophylactically. The mouth should be rinsed out for a few minutes three or four times daily.

The appearance of a bismuth line is, in my experience, 


\section{DENTAL DISEASE}

usually associated with concomitant dental disease. This is borne out by the figures already quoted-only 7 per cent. of the dentally "fit" group developed a bismuth line (very slight in all cases), compared with 75 per cent. of the "unfit" group, in whom the condition was frequently more severe.

Should a true bismuth stomatitis arise, the administration of bismuth should be discontinued at once and attention directed to the correction of the causal factor.

Pending the treatment of the dental lesion the patient should be instructed to use a mouthwash very frequently (hourly, if possible). The advantages of common salt are again apparent, and it is as equally effective therapeutically as prophylactically. Brushing of the teeth and gums during the acute stage of a true bismuth stomatitis is, in the author's opinion, inadvisable. Intravenous administration of calcium thiosulphate in severe cases frequently effects rapid symptomatic improvement. The initial dose is $0.6 \mathrm{gm}$., followed the next day by $0.9 \mathrm{gm}$., and continued daily for three to seven days as required.

Treatment with bismuth should not be recommenced for some time after the dental condition has returned to normal, and then only under careful observation.

\section{Summary AND Conclusions}

The results of dental examination of 400 unselected, consecutive syphilitic patients, along with 400 nonsyphilitic " controls" are given.

The results show that there is no material difference in the basic incidence of dental lesions in the syphilitic and non-syphilitic groups.

The importance of dental sepsis as a causal factor in the mechanism of intolerance to antispecific therapy is emphasised.

The most common complication of modern treatment is a bismuth line ; this occurred in 75 per cent. of patients with unhealthy mouths and in only 7 per cent. of patients with healthy mouths. The former group suffered interruption of treatment for periods varying from two to fourteen weeks, while in no case in the "fit " group had bismuth administration to be discontinued.

The importance of dental hygiene during antisyphilitic 287 


\section{BRITISH JOURNAL OF VENEREAL DISEASES}

therapy and the necessity for co-operation between the dental profession and the V.D. clinicians in the treatment of any existing dental lesions are urged.

I have to thank Mr. R. C. L. Batchelor, Surgeon in charge of Venereal Diseases, Edinburgh Royal Infirmary, for access to clinical material under his charge, and to Dr. A. E. W. McLachlan for facilities for clinical investigations in the Joint Committee's Clinic, Newcastle-uponTyne.

\section{REFERENCES}

(I) Azoulay, R. (r922): Press med., 30, I34.

(2) Buschke, A. (I924): Zentralbl. f. Haut. u. Geschlechtkr., 11, 9.

(3) McLachlan, A. E. W. (I937) : J. Chemotherapy, 12 , 127.

(4) Milian and Perin (I922): Bull. et mem. Soc. de dermat. et syph.,

(5) Milian and Perin (I922): Bull. et mém. Soc. med. d. Hôp. de Par., 38, I35.

(6) Stokes, J. H., and Cathcart, E. P. (r923) : Arch. Dermat. and Syph., 7, I4. 